\title{
The usage of infrared thermography to investigate spatial variations of heat transfer at spray cooling
}

\author{
Anton Surtaev ${ }^{1,2 *}$, Aleksandr Nazarov ${ }^{1}$, Anatoliy Serov ${ }^{1}$, Nikolay Miskiv ${ }^{1}$, \\ and Vladimir Serdyukov ${ }^{1,2}$ \\ ${ }^{1}$ Kutateladze Institute of Thermophysics SB RAS, Lavrentyev ave. 1, 630090 Novosibirsk, Russia \\ ${ }^{2}$ Novosibirsk State University, 2 Pirogova str., 630090 Novosibirsk, Russia
}

\begin{abstract}
In present paper new approach to study heat transfer at spray cooling, based on the using of high-speed infrared thermography with high spatial resolution is proposed. Also in the paper new data on basic spray parameters, including sizes and velocities of droplets at different pressure at the nozzle inlet were obtained with the use of shadow technique and high-speed video camera. It is found, that heat transfer coefficient is unequally spatially distributed value and essentially depends on flow rate in the stationary irrigation mode. The dependence of heat transfer coefficient on a distance between spray source and heat exchange surface is obtained and an optimal distance corresponding to the maximum heat transfer intensity at present configuration of irrigation points relatively to the heating surface is determined.
\end{abstract}

\section{Introduction}

Spray cooling as well as micro/mini heat pipes and jet impingement cooling is one of the most effective ways for heat dissipation; therefore nowadays this direction receives much attention [1-3]. Despite all advantages of spray cooling this process is still complicated for physical description. In particular, heat transfer at spray cooling depends on many factors, including the flow rate, the distance from the spray source to the target, the sizes and velocities of droplets, dynamic characteristics of the intermittent spray, etc. At the same time, previous studies on heat transfer are based on measuring the local surface temperature, generally with the use of thermocouples with low temporal and spatial resolutions. According to these measurements the integral heat transfer coefficient was estimated for the entire heat transfer surface. In reality, heat transfer coefficient is unequally distributed over a heat exchange surface [4], and is non-stationary value, especially under conditions of a intermittent spray. Therefore, for better understanding and subsequent interpretation of observed phenomena, it is necessary to measure the spatial distribution of the heat transfer coefficient over time at spray cooling.

Present paper provides an illustration of how to investigate the spatial distribution of heat transfer coefficient with the use of high-speed infrared (IR) thermography. Also, for

* Corresponding author: surtaevas@gmail.com 
the future analysis of the influence of key hydrodynamic parameters of spray on heat transfer, new data on droplets sizes, their velocity, spray angle using the shadow technique and high-speed visualization were obtained in the study.

\section{Experimental setup}

To investigate hydrodynamic parameters of the spray and heat transfer under stationary and pulsed irrigation modes, a specially designed experimental setup was used. Four Bosch EV14L nozzles were used as a spray source. The description of the spray generation system and the nozzle layout scheme are presented in [5]. As a target, $400 \mu \mathrm{m}$ thick sapphire substrate was used, on the back side of which a thin film heater from indium tin oxide (ITO) with $1 \mu \mathrm{m}$ thickness and area of $S_{\text {area }}=4 \mathrm{~cm}^{2}$ was deposited. The main advantage of using ITO as a heater material in experiments at spray cooling is its transparency in the visible spectrum $(380-750 \mathrm{~nm})$ and opacity in the mid-IR spectrum $(3-5 \mu \mathrm{m})$. This property makes it possible to measure non-stationary temperature field on the ITO film surface by infrared camera and visually record the dynamics of irrigation of heat transfer surface by high-speed video camera. Therefore, such construction of the heating element was recently widely used for pool boiling experiments [6-8]. The substrate with the thin film heater was mounted on plexiglas frame. The spray source with nozzles and the frame with the target were mounted on moving platforms located on the optical rail, which made it possible to vary the distance from the spray source to the target in the range of 55 - 395 $\mathrm{mm}$. Distilled water was used as a working fluid, the initial liquid temperature was similar to room temperature and varied in the range of $15-24{ }^{\circ} \mathrm{C}$. The fluid flow rate $Q$ for the stationary spray was varied in the range of $5.3-8.2 \mathrm{~cm}^{3} / \mathrm{s}$ by controlling the pressure drop $(\Delta p)$ at the nozzle inlet in the range of $1.5-3.5$ bar.

Since the intensity of heat transfer at spray cooling depend on such spray parameters, as the size and velocity distributions of droplets, in the first stage the following experiment was carried out. A high-speed video camera was installed from the side of the spray source opposite to the light source. The visualization by the shadow method was carried out with a frequency of up to $10 \cdot 10^{3}$ FPS and with a maximum resolution of $40 \mu \mathrm{m}$. To analyze the irrigation pattern of the base surface, high-speed visualization was also performed on the reverse side of the transparent heat exchange surface with a recording frequency of up to $2 \cdot 10^{3}$ FPS.

To measure the temperature field of the irrigated surface, high-speed IR camera was installed behind of the target. IR recording was carried out with frequency of 200 FPS and resolution of $88 \mu \mathrm{m}$. Before the experiments, calibration procedure of IR recording was performed, in which conditions of uniform surface superheating were created. The temperature in these experiments was monitored using precision platinum temperature sensor. As the result, the error of temperature measurement using IR camera did not exceed $1{ }^{\circ} \mathrm{C}$. Sample was resistively heated (Joule heating) by a DC power supply via silver electrodes deposited onto the ITO film. The heat flux density was calculated as $q=V \cdot I / S_{\text {area }}$. The error in measuring the heat flux density taking into account heat losses does not exceed $5 \%$.

\section{Results}

It is known that the heat transfer intensity in non boiling regime at spray cooling depend on droplet sizes, their velocity and irrigation pattern of the impact surface [4], therefore, at the first stage, data obtained by the shadow method were analyzed. The images (Fig. 1a) show the propagation of the jets front from a single nozzle with four holes at intermittent spray 
mode ( $\Delta p=2$ bar). As it can be seen from these images, such visualization allows us to determine the whole range of key hydrodynamic parameters with high accuracy. In Fig. 1b the droplet size distribution at given distance from the nozzle plane $(z=20 \mathrm{~mm})$ is shown. The measurements were carried out at the distance exceeding distance of the instability development and the jet disintegration into individual drops. The analysis shows that the deviation of droplet sizes from the most probable value does not exceed $0.13 \mathrm{~mm}$ with an average size of droplets in the range of $0.4-0.55 \mathrm{~mm}$. Processing the entire array of droplet size data shows that the average droplet size decreases slightly with increase in pressure drop. Analysis of data on droplets velocity shows that the variation of average velocity value, depending on droplets size, does not exceed $2 \%$. At the same time, the droplet velocity significantly depends on pressure and reaches a value of $U=18.5 \mathrm{~m} / \mathrm{s}$ at $Q=8.2$ $\mathrm{cm}^{3} / \mathrm{s}(\Delta p=3.5$ bar $)$.
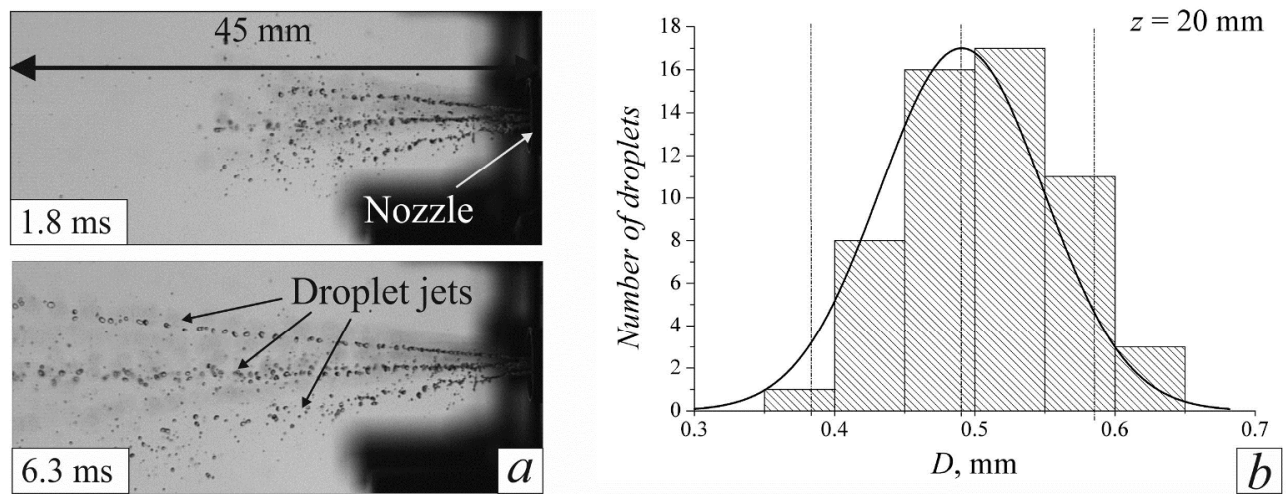

Fig. 1. a) The frames of high-speed visualization of the droplet jets coming from one nozzle $(\Delta p=2$ bar); $b$ ) Droplets size distribution $(\Delta p=2$ bar, $U=11.5 \mathrm{~m} / \mathrm{s}, z=20 \mathrm{~mm}, z$ - distance from nozzle to measurement point).

As the analysis showed, the spray angle of droplet jets is $6-7^{\circ}$ and does not change with increase in pressure drop in investigated range. Also, the implemented symmetry of the droplet jets allows estimating the area of impacted surface depending on the distance from the spray source to the target, including in the multi-nozzle configuration. The resulting array of experimental data will be used in the future work both to develop the optimal configuration of nozzles for cooling a given heat exchange surface, and to analyze the characteristics of heat transfer at spray cooling.

At the next step, the visualization data on the character of irrigation of impacted surface, as well as the temperature field of the heater at various flow rates of a stationary spray depending on the distance from the source to the target and the heat flux density up to 254 $\mathrm{kW} / \mathrm{m}^{2}$ were analyzed. As it can be seen from the high-speed video frames (Fig. 2a) recorded from the backside of the surface impacted by droplets under adiabatic conditions, such visualization allows us to identify target areas with maximum irrigation degree, wetted and dry areas, and also to identify drops impacting on the surface of a liquid film, formed on the target. In particular, data show that with increasing distance from the spray source to the target in the range of $55-155 \mathrm{~mm}$, flux density of the impacted droplets increases. These conclusions are based on estimations of number of concentric rings (indicated by arrows in Fig. 2a) per time unit, arising at the moment of the collision of droplets with the liquid film. 

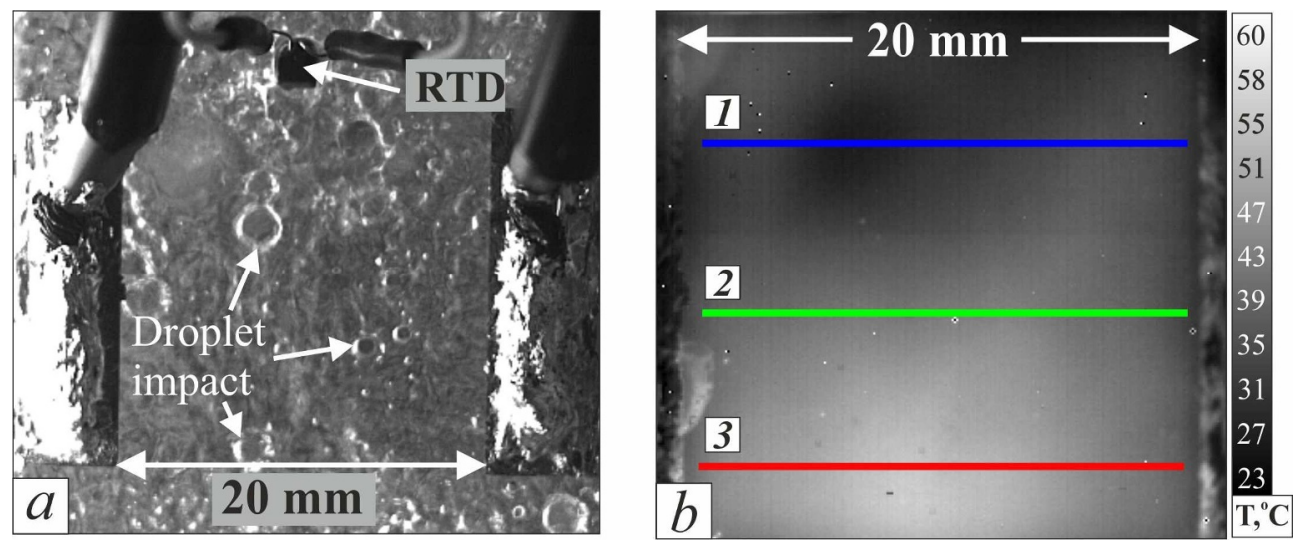

Fig. 2. a) The frame of high speed-visualization from the backside of impacted surface at stationary spray irrigation ( $\triangle p=2$ bar, $\left.l=125 \mathrm{~mm}, q=0 \mathrm{~W} / \mathrm{m}^{2}\right) ; b$ ) Temperature field of the heater surface $\left(\Delta p=2\right.$ bar, $\left.l=155 \mathrm{~mm}, q=154.7 \mathrm{~kW} / \mathrm{m}^{2}\right)$.

Fig. $2 b$ presents the frame of IR recording of the ITO heater at heat flux density of $q=154.7 \mathrm{~kW} / \mathrm{m}^{2}$. Analysis of the time-averaged temperature field shows that there are zones with different surface temperatures. In particular, from the frame it can be clearly seen the presence of concentric "dark" area with the lowest temperature, which is corresponds to the impact area of droplet jet. In the bottom part, on the contrary, the temperature is noticeably higher than in the upper part of the heater. These conclusions are confirmed by the graph (Fig. 3a), which shows the temperature distribution of the surface across the width of the heater for different coordinates along the gravity force. As it can be seen from the bottom curve (blue color), the temperature in the region of jet impact may differ by $9{ }^{\circ} \mathrm{C}$ from the temperature of the heater in another region at given distance from the upper edge of the heater.

An analysis of the non-stationary temperature field also indicates that in some areas temperature fluctuations are observed, apparently related to a local enhancement of heat transfer due to impact of the individual drops on the liquid film surface. However, these pulsations are quite difficult to identify due to the relatively low frequency of the IR recording and, first of all, to the fact that the temperature field was measured not directly on the impacted surface. Certainly, performing IR recording in the case when the spray irrigates directly a thin-film heater (as, for example, it was done in boiling experiments $[6,8]$ ), would significantly improve the measurement of the amplitude-frequency characteristics of temperature fluctuations of a heater surface. Therefore, the analysis of the effect of individual liquid droplets impact on film surface on the local and integral heat transfer at spray cooling is one of the tasks of future research.

Using of IR recording, as it was shown by the primary data processing, provides an incredibly wide range of information for analysis. At the present stage, authors decided to focus on studying the effect of the distance from a spray source to a target on integral heat transfer. For this, series of experiments was carried out in which IR recording was performed for a given heat flux $\left(q=79 \mathrm{~kW} / \mathrm{m}^{2}\right)$ and fluid flow rate $\left(Q=6.6 \mathrm{~cm}^{3} / \mathrm{s}\right)$ with a distance change in the range of $55-395 \mathrm{~mm}$ with a step of $20 \mathrm{~mm}$. During data processing, temperature field data were averaged over the time $(10 \mathrm{sec})$ and the total area of the heater. 

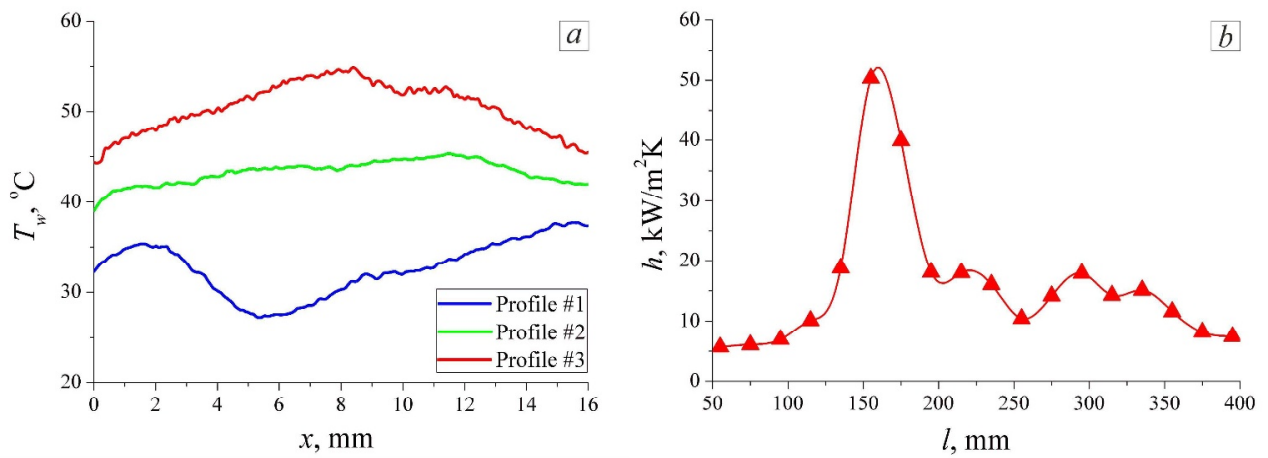

Fig. 3. a) Heating surface temperature distribution along the lines, showed in Fig. $2 \mathrm{~b}$ ( $\Delta p=2$ bar, $\left.l=155 \mathrm{~mm}, q=154.7 \mathrm{~kW} / \mathrm{m}^{2}\right) ; b$ ) dependence of heat transfer coefficient on the distance from the spray source to the impacted surface target at stationary irrigation $\left(\Delta p=2\right.$ bar, $\left.q=79 \mathrm{~kW} / \mathrm{m}^{2}\right)$.

In Fig. $3 b$ the dependence of heat transfer coefficient $\left(h=q /\left(T_{h}-T_{0}\right)\right.$, where $T_{h}$ is the ITO film surface temperature, $T_{0}$ is the liquid temperature in the nozzle inlet) on the distance from the spray source to the target $(l)$ is presented. The graph shows that the smallest value of the heat transfer coefficient with given configuration of irrigation points relative to heater is observed conditionally for distances up to $100 \mathrm{~mm}$ and after $350 \mathrm{~mm}$. As the analysis of high-speed video data showed, this is due to the fact that at small distances from spray source to target, the areas of droplet jets impact are located around the perimeter of the heating surface and only partially irrigate the heat release area. The decrease in heat transfer coefficient at large distances $(l>350 \mathrm{~mm})$ is related to the fact that over a long distance the irrigation area increases (since the spray angle is not zero), which invariably leads to a decrease in the local flow rate of the liquid on the base surface. Also from the graph presented in Fig. $3 \mathrm{~b}$ it can be seen that there is the certain distance $l \sim 155-160 \mathrm{~mm}$, at which the maximum heat transfer coefficient is observed with value in 10 times more than the heat transfer intensity for $l<100 \mathrm{~mm}$ and $l>350 \mathrm{~mm}$. Here it should be noted that obtained result would not change with an increase in the number of irrigation points at their given configuration at the spray source. At the same time, it seems that a change in the configuration of the nozzles relative to the heat exchange surface could lead to a change in the integral heat transfer and its dependence on the distance between spray source and target, especially at small distances of $l<250 \mathrm{~mm}$.

\section{Conclusions}

In the present study experimental setup with integrated high-speed methods was created, which allows to measure both the main hydrodynamic parameters of the spray and spatial distribution of the temperature field of the heat transfer surface with the ability to control the spray irrigation regimes. New experimental data on the droplets sizes, their velocity and the spray angle, depending on the pressure drop were obtained. It has been shown that highspeed video recording from the backside of transparent heater allows to identify the irrigation pattern with high accuracy, e.g. to measure the area of the spray cone, impact areas of the droplet jets and liquid film on the heat exchange surface, as well as to measure the density of the droplets falling on the liquid film surface. It has been demonstrated that the heat transfer coefficient in non boiling regime is unequally spatially distributed value over the heater surface. For example, the temperature in the region of the impact droplet jet is much lower than in other areas of the heater. It is shown that the heat transfer coefficient depends on the distance from the spray source to the heating surface during stationary irrigation. The optimal distance with the maximum heat transfer coefficient has been 
determined for a given configuration of irrigation points relative to the heating surface of a given area. However, authors would like to emphasize that this study is only the beginning of the path in understanding the mechanisms of heat transfer at spray cooling and their subsequent description through the use of modern high-speed experimental methods.

The reported research was funded by Russian Foundation for Basic Research and the government of the Novosibirsk region of the Russian Federation, grant № 18-48-540018.

\section{References}

1. N. Zhou, F. Chen, Y. Cao, M. Chen, Y. Wang, Appl. Therm. Eng. 112 (2017)

2. W. Zhang, W. Zhaoliang, Exp. Therm. Fluid Science 69 (2015)

3. M.R.O. Panão, A.L.N. Moreira, D.F.G. Diamantino, Int. J. of Eng. and Industrial Management 3 (2015)

4. G. Liang, I. Mudawar, Int. J. of Heat and Mass Transf., 115 (2017)

5. A.D. Nazarov, A.F. Serov, V.I. Terekhov, K.A. Sharov, J. of Eng. Phys. and Thermophys. 82(6) (2009)

6. V.S. Serdyukov, A.S. Surtaev, A.N. Pavlenko, A. N. Chernyavskiy, High Temperature 56(4) (2018)

7. J. Petkovsek, Y. Heng, M. Zupancic, H. Gjerkes, F. Cimerman, I. Golobic, Int. J. of Refrigeration 61 (2016)

8. S. Jung, H. Kim, Heat Transf. Eng. 36(12) (2015). 